\title{
Management of screening-detected stage I lung cancer
}

\author{
Raymond U. Osarogiagbon \\ Thoracic Oncology Research Group, Multidisciplinary Thoracic Oncology Program, Baptist Cancer Center, Memphis, TN 38120 , USA \\ Correspondence to: Raymond U. Osarogiagbon, MBBS, FACP. Thoracic Oncology Research Group, Multidisciplinary Thoracic Oncology Program, \\ Baptist Cancer Center, 80 Humphreys Center Drive, Suite 220, Memphis, TN 38120, USA. Email: rosarogi@bmhcc.org.
}

Submitted Sep 19, 2016. Accepted for publication Sep 27, 2016.

doi: $10.21037 /$ jtd.2016.10.78

View this article at: http://dx.doi.org/10.21037/jtd.2016.10.78

Easily the biggest development in the fight against lung cancer since the 1964 US Surgeon General's report directly linked tobacco use to lung cancer, is the emergence of high-quality data clearly establishing the efficacy and costeffectiveness of computed tomographic (CT)-screening as a means of improving lung cancer survival (1-4). Only tobacco control for primary prevention can have greater populationlevel impact on lung cancer mortality and survival statistics (5). Given the tight link between stage at treatment and survival, CT screening, by allowing early identification, leads to high lung cancer cure rates (3). Participation in screening is also a tremendous teachable moment that can potentially enhance smoking cessation, thereby contributing to primary (and secondary) prevention.

As great as the opportunity presented by CT screening, is the challenge of implementation. Translating the efficacy of CT screening in relatively controlled environments to realworld effectiveness will be a decades-long challenge. Part of this challenge will be determining the optimal management of screening-detected lung cancer, the objective being to limit treatment-related morbidity and mortality, while preserving benefit. For full benefit, optimal treatment must accompany screening-detected lung cancer. Part of the challenge is understanding how the biology of screeningdetected lung cancer is similar to, and different from, lung cancer serendipitously detected on imaging studies done for unrelated reasons, and lung cancer detected on the basis of clinical symptoms.

Currently, the Tumor-Node-Metastasis (TNM) staging system is our best prognostic and treatment guide. This system, which continues to undergo modification as knowledge expands, has mostly been developed in lung cancer detected serendipitously in unselected patients, and during evaluation of clinically symptomatic patients. The extent to which the prognostic parameters of the TNM system apply to screened populations, especially those at the earliest of stages, and therefore, the approach to risk mitigation during treatment, should be an area of great interest (6). For example, the thoroughness of application of nodal staging is the major quality of care deficit in conventionally diagnosed and treated lung cancer patients, associated with the greatest survival disparity, especially in potentially curable, early stage non-small cell lung cancer (7-12). Multiple investigators have demonstrated the depth, and breadth, of the suboptimal nodal staging problem in surgical and non-surgical patients, ranging from the use of radiologic and invasive nodal staging tests, through to the surgical retrieval and pathologic evaluation of directly and indirectly retrieved lymph nodes in resection specimens (7-17).

Are these concerns relevant to screening-detected early stage patients, or are there subsets of such patients for whom the rules of surgical and non-surgical staging (and treatment) need to be modified because of biologic differences in screening-detected lung cancer? There may be an opportunity to tailor management to patient risk and temper the rules for risk-mitigation in the screen-detected population. Which patients can dispense with invasive and non-invasive staging procedures? What about surgical versus non-surgical treatment options: are early screeningdetected patients potentially candidates for non-surgical treatments, thereby avoiding the morbidity and mortality risk of surgical intervention? When surgery is chosen, can we limit the extent of surgical resection, thereby preserving functional lung volume and limiting long-term morbidity, thus improving postoperative quality of life? Can we heed the lesson of breast cancer, in which the use of lumpectomy has superseded mastectomy, an evolution accelerated by mammographic detect of tumors at much earlier stage? Can 
we dispense with hilar and mediastinal nodal examination in certain early stage screening-detected lung cancer patients, again heeding the lesson of breast cancer (18)? Bluntly put, are there patients who can do without nodal examination, without extensive resection, without surgery?

The recent publication by Flores et al. in the Annals of Surgery, addresses the question of nodal examination (19). In a retrospective analysis of the International Early Lung Cancer Action Program (I-ELCAP) database of CT screening-detected lung cancer from 1992 to 2014, limited to patients with resected clinical T1a/b N0M0 non-small cell lung cancer, they identified 462 patients with, and 145 patients without, mediastinal lymph node resection (MLNR). In further analyses, they separated the cohort into those whose cancer manifested as a sub-solid nodule (151 vs. 52 patients) and those with a solid nodule (311 vs. 93 patients). They compared certain demographic, disease and treatment characteristics as well as long-term survival between those with and without MLNR. They found similar survival between those who had and did not have MLNR: $92 \%$ vs. $96 \%(\mathrm{P}=0.19)$ for the whole group; $100 \%$ vs. $100 \%$ for those with subsolid nodules; $87 \%$ vs. 94\% $(\mathrm{P}=0.24)$ for those with solid nodules. They identified advancing age, central tumor location, tumor size $>20 \mathrm{~mm}$, and invasion beyond the lung stroma as independent risk factors for death (19). They conclude that MLNR is not mandatory when screening-diagnosed NSCLC manifests as a sub-solid nodule.

The ELCAP and I-ELCAP group have performed tremendous, ground-laying work in the lung cancer screening arena, prior to the National Lung Screening Trial $(1,2)$. Despite initial criticism of their uncontrolled prospective observational study design and concerns about the dangers of lead-time, length and over-diagnosis bias, the randomized NLST results have largely validated their early findings on the value of screening CT $(3,4,20,21)$. IELCAP's prospective multi-institutional cohort has the advantages of rigorous data definition, standardized data collection and end-point ascertainment practices, and a long duration of follow up. However, the current report is a retrospective analysis, with all the limitations inherent in such analyses. As with the NLST, there is no prespecification for cancer treatment (where the rubber meets the road), but rather a pragmatic approach is used, in which treatment decisions are left to the managing clinicians. This methodologic approach has the advantage of potentially improved external validity (generalizability to 'real-world' populations), but a disadvantage of weakened internal validity, especially in the comparability of ad-hoc internal cohorts, such as in the analyses by Flores et al. Additional potential sources of bias are the absence of information on use of invasive preoperative nodal staging (how much preoperative invasive nodal staging did the cohorts receive?). There was no separation between operative mortality and cancer mortality: the investigators (by their admission) have conflated all deaths resulting from treatment as lung cancer deaths, making it difficult to evaluate the challenges of surviving the cancer versus surviving the surgery.

Illustrating the inherent biases of this study design, by their reported demographic and clinical comparisons, the MLNR population appears to be at higher risk than the no MLNR cohort in terms of central location, smoking history, and tumor size. No surprise then that that group's outcomes seem to be worse. One could speculate that the outcome difference might have been greater without the added information from the nodal dissection, given the additional detection of more advanced pathologic stage than initially suspected, and the likely post-operative management response triggered by detection of nodal metastasis in more contemporary patients in the era of beneficial adjuvant therapies. Whilst it is impossible to know if the 'no MLNR' cohort had similar nodal involvement, the suggestion is probably not, given the paucity of hilar/intrapulmonary nodal metastasis (assuming relatively similar patterns of lymph node gross dissection in the provided parenchymal resection specimens), although the higher rate of sublobar resection (with its likely impact on nodal examination) makes even this unknowable.

It is instructive that even in this ostensibly low risk population, $11 \%$ of patients with a solid nodule who had MLNR, all of whom were clinically node-negative, had unexpected pathologic nodal metastasis, including $6 \%$ with N2 disease. Similar data on the patients with sub-solid nodules are not reported. This is not reassuring, as we have previously pointed out (22). At this time, a recommendation against pathologic nodal evaluation seems akin to opening Pandora's box: the unreported procedures for selection between the MLNR and no-MLNR approaches that directed the I-ELCAP surgeons will not be communicated in general practice. All the average practitioner will hear is that 'MLNR is unnecessary', without communication of the nuances of the decision-making involved in careful selection of such a strategy, and, worse, without prospective evaluation of the soundness of such selection strategies. We have seen out of Pandora's box, the wrong interpretation of ACOSOG Z0030, which some have erroneously cited 
as license to perform suboptimal nodal dissection. Finally, minor pedantry: it is unclear why Flores et al. have referred to lesions $>20 \mathrm{~mm}$ as T1a. These are pT1b, although in the absence of nodal or other metastases the aggregate stage remains IA, but that is not the same thing...

The above reservations notwithstanding, the report by Flores et al. provides great food for thought. It is almost certain that nodal examination, extensive resection and (albeit well beyond the scope of this report) even surgical intervention, are not necessary in all patients with lung cancer. This hypothesis-generating report reminds us of the need to systematically evaluate which patients can dispense with more morbid processes of care delivery. A reasonable place to start this inquest is in screening-detected patients with small sub-solid nodules or ground-glass opacities. However, more rigorously controlled, prospective studies are needed to validate any recommended practice change. This report should provide greater equipoise to clinical investigators, allowing them to overcome ethical concerns that inhibit enrollment of patients into ongoing prospective trials that seek to help interrogate each of the three clinical questions: the minimum oncologically sound extent of pulmonary parenchymal resection; the extent of nodal evaluation; and the use of surgical $v s$. non-surgical treatment modalities.

In the absence of results from such studies, it is important to remain aware of the limitations of ad-hoc retrospective analyses. Let us bear in mind the paradox of retrospective timeliness of care studies, which consistently show that recipients of delayed lung cancer care have significantly better survival than recipients of timely care (23-25). Clearly, the conclusion from such studies cannot be that delaying delivery of care for a lethal disease such as lung cancer leads to better survival. Consequently, these studies have not led to recommendations for postponing definitive care for lung cancer. Rather, the results are widely acknowledged to reflect the existence of overwhelming and uncorrected asymmetry in the comparative populations.

\section{Acknowledgements}

The study was supported by NIH R01 CA172253.

\section{Footnote}

Provenance: This is an invited Commentary commissioned by the Section Editor Feichao Bao (Department of Thoracic Surgery, the First Affiliated Hospital, Zhejiang University,
Hangzhou, China).

Conflicts of Interest: The author has no conflicts of interest to declare.

Comment on: Flores RM, Nicastri D, Bauer T, et al. Computed Tomography Screening for Lung Cancer: Mediastinal Lymph Node Resection in Stage IA Nonsmall Cell Lung Cancer Manifesting as Subsolid and Solid Nodules. Ann Surg 2016. [Epub ahead of print].

\section{References}

1. Henschke CI, McCauley DI, Yankelevitz DF, et al. Early Lung Cancer Action Project: overall design and findings from baseline screening. Lancet 1999;354:99-105.

2. International Early Lung Cancer Action Program Investigators, Henschke CI, Yankelevitz DF, et al. Survival of patients with stage I lung cancer detected on CT screening. N Engl J Med 2006;355:1763-71.

3. National Lung Screening Trial Research Team, Aberle DR, Adams AM, et al. Reduced lung-cancer mortality with low-dose computed tomographic screening. $\mathrm{N}$ Engl J Med 2011;365:395-409.

4. Black WC, Gareen IF, Soneji SS, et al. Cost-effectiveness of CT screening in the National Lung Screening Trial. N Engl J Med 2014;371:1793-802.

5. Osarogiagbon RU. Editorial preface to this special issue "Improving the quality and outcomes of lung cancer care: an interdisciplinary approach". Transl Lung Cancer Res 2015;4:322-6.

6. Patz EF Jr, Pinsky P, Gatsonis C, et al. Overdiagnosis in low-dose computed tomography screening for lung cancer. JAMA Intern Med 2014;174:269-74.

7. Farjah F, Flum DR, Ramsey SD, et al. Multi-modality mediastinal staging for lung cancer among medicare beneficiaries. J Thorac Oncol 2009;4:355-63.

8. Detterbeck F. What is quality and does it matter? J Thorac Oncol 2009;4:279-80.

9. Detterbeck F, Puchalski J, Rubinowitz A, et al. Classification of the thoroughness of mediastinal staging of lung cancer. Chest 2010;137:436-42.

10. Osarogiagbon RU, Yu X. Mediastinal lymph node examination and survival in resected early-stage non-smallcell lung cancer in the surveillance, epidemiology, and end results database. J Thorac Oncol 2012;7:1798-806.

11. Osarogiagbon RU, Yu X. Nonexamination of lymph nodes and survival after resection of non-small cell lung cancer. Ann Thorac Surg 2013;96:1178-89. 
12. Osarogiagbon RU, Darling GE. Towards optimal pathologic staging of resectable non-small cell lung cancer. Transl Lung Cancer Res 2013;2:364-71.

13. Little AG, Rusch VW, Bonner JA, et al. Patterns of surgical care of lung cancer patients. Ann Thorac Surg 2005;80:2051-6; discussion 2056.

14. Ludwig MS, Goodman M, Miller DL, et al. Postoperative survival and the number of lymph nodes sampled during resection of node-negative non-small cell lung cancer. Chest 2005;128:1545-50.

15. Smeltzer MP, Faris N, Yu X, et al. Missed Intrapulmonary Lymph Node Metastasis and Survival After Resection of Non-Small Cell Lung Cancer. Ann Thorac Surg 2016;102:448-53.

16. Osarogiagbon RU, Ogbata O, Yu X. Number of lymph nodes associated with maximal reduction of long-term mortality risk in pathologic node-negative non-small cell lung cancer. Ann Thorac Surg 2014;97:385-93.

17. Osarogiagbon RU, Decker PA, Ballman K, et al. Survival Implications of Variation in the Thoroughness of Pathologic Lymph Node Examination in American College of Surgeons Oncology Group Z0030 (Alliance). Ann Thorac Surg 2016;102:363-9.

18. Giuliano AE, Hunt KK, Ballman KV, et al. Axillary dissection vs no axillary dissection in women with invasive

Cite this article as: Osarogiagbon RU. Management of screening-detected stage I lung cancer. J Thorac Dis 2016;8(10):E1416-E1419. doi: 10.21037/jtd.2016.10.78 breast cancer and sentinel node metastasis: a randomized clinical trial. JAMA 2011;305:569-75.

19. Flores RM, Nicastri D, Bauer T, et al. Computed Tomography Screening for Lung Cancer: Mediastinal Lymph Node Resection in Stage IA Nonsmall Cell Lung Cancer Manifesting as Subsolid and Solid Nodules. Ann Surg 2016. [Epub ahead of print].

20. Twombly R. Lung cancer screening debate continues despite international CT study results. J Natl Cancer Inst 2007;99:190-5.

21. Patz EF Jr, Goodman PC, Bepler G. Screening for lung cancer. N Engl J Med 2000;343:1627-33.

22. Osarogiagbon RU, Rami-Porta R. Early stage nonsmall-cell lung cancer: surgical implications of the new adenocarcinoma classification. J Thorac Oncol 2013;8:e45-6.

23. Myrdal G, Lambe M, Hillerdal G, et al. Effect of delays on prognosis in patients with non-small cell lung cancer. Thorax 2004;59:45-9.

24. Gould MK. Delays in lung cancer care: time to improve. J Thorac Oncol 2009;4:1303-4.

25. Koo MM, Zhou Y, Lyratzopoulos G. Delays in diagnosis and treatment of lung cancer: Lessons from US healthcare settings. Cancer Epidemiol 2015;39:1145-7. 